\title{
The pregnancy cycle approach to safe motherhood
}

\author{
Saumya RamaRao \\ Population Council \\ Sarah Raifman \\ Population Council
}

Follow this and additional works at: https://knowledgecommons.popcouncil.org/departments_sbsr-rh

Part of the International Public Health Commons, Maternal and Child Health Commons, Obstetrics and Gynecology Commons, Public Health Education and Promotion Commons, Social and Behavioral Sciences Commons, and the Women's Health Commons How does access to this work benefit you? Let us know!

\section{Recommended Citation}

RamaRao, Saumya and Sarah Raifman. 2010. "The pregnancy cycle approach to safe motherhood," brief. Washington, DC: Population Council. 


\section{(P Population Council

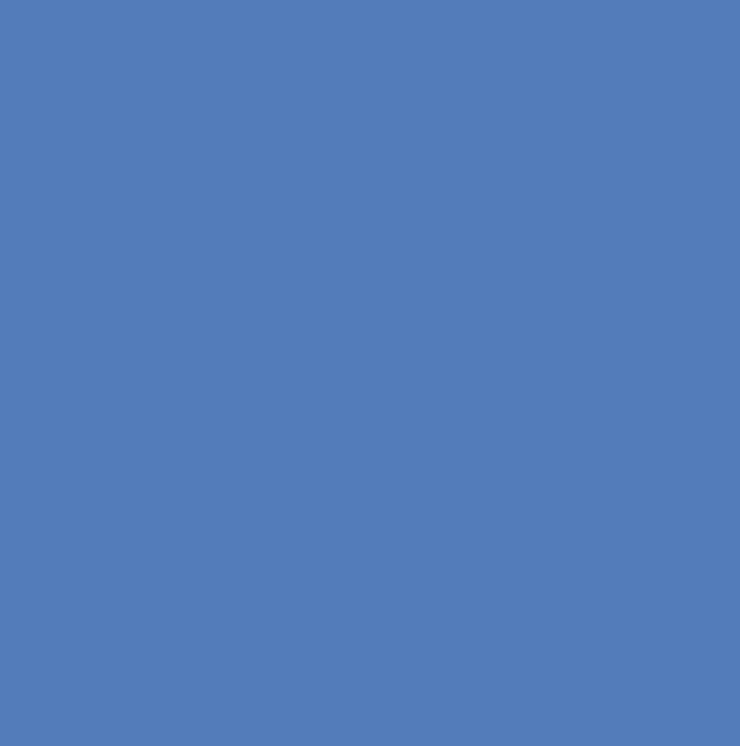

www.popcouncil.org

4301 Connecticut Ave, NW
Suite 280
Washington, DC 20008

4301 Connecticut Ave, NV
Suite 280
Washington, DC 20008

4301 Connecticut Ave, NW
Suite 280
Washington, DC 20008

Tel: (202) 237-9400
Tel: (202) 237-9400

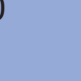

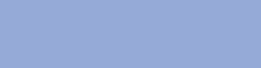




\section{Contents}

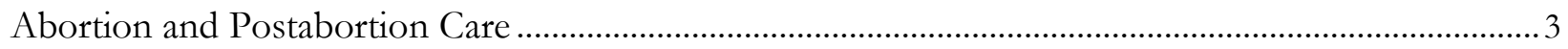

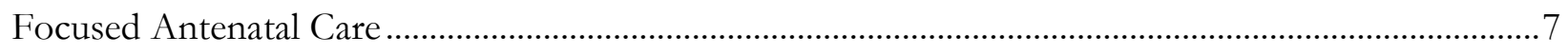

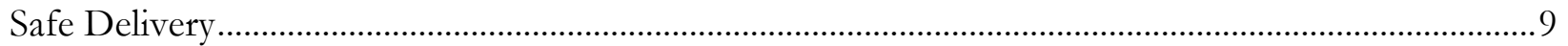

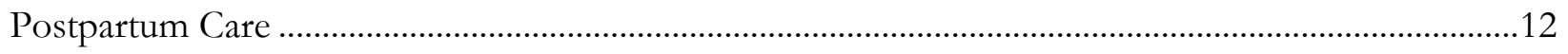

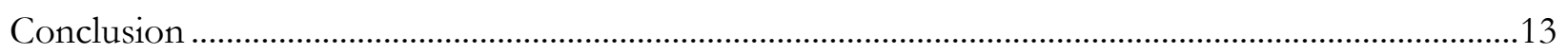




\section{Introduction}

The Population Council's Reproductive Health program aims to address current public health challenges by generating evidence, developing and testing new technologies, and working in partnership with key stakeholders in the public and private sectors. The aim is to contribute to the UN Millennium Development Goals and the reproductive well-being and health of men and women globally.

Particular emphases for the program are MDG4 and MDG5. The program has adopted a pregnancy cycle approach whereby women's needs from facilitating a desired pregnancy to successful parturition are addressed in a range of settings - home, community and health facility. This approach is aligned with the "continuum of care" approach so as to leverage policy and program opportunities as they rise. Furthermore, it aims to contribute to both MDG4 and MDG5 by focusing on the mother-baby dyad since their individual health outcomes are closely interlinked.

In recognizing the immensity and scope of the challenge, the Council's RH program partners with other key stakeholders such as the Maternal Health Task Force (MHTF), the Partnership for Maternal, Newborn and Child Health (PMNCH), and the PAC Consortium. The Council contributes technical assistance, Operations Research capabilities for testing innovative models, $M \& E$ expertise and methodical documentation. By testing innovations and facilitating the scale-up of evidence-based practices, we hope to contribute to improved maternal and child health.

In the following four sections, the document will address different stages of the pregnancy cycle: abortion and postabortion care, antenatal care, delivery, and postpartum and beyond.

\section{Abortion and Postabortion Care}

\section{Research}

Population Council research aims to save women's lives by reducing the morbidity and mortality caused by unsafe abortion and lack of postabortion care and, in countries where abortion is legal, by making services safer. Poor quality of medical care, substandard provider-client communication, and lack of counseling on medical procedures and recuperation are just some of the problems experienced by women with complications from miscarriage and incomplete abortion. In addition, scant attention too often is paid to patients' other health concerns_-including their desire for effective and affordable contraception.

Population Council research on postabortion care and abortion has been conducted primarily through the Council's FRONTIERS and Ebert programs, respectively. They include clinical studies for the development of new technology, and Operations Research to test service-delivery and feasibility, as well as policy work to promote the integration of PAC through involvement in the 
Postabortion Care Consortium and USAID's PAC Connect. The Council's research on abortion involves a range of community- and clinic-based efforts focusing on improving the safety of abortion services in settings in which abortion is legal; testing innovative ways to measure prevalence rates; exploring determinants of abortion and care-seeking behavior; and investigating abortion knowledge and attitudes among such groups as clients, men, and providers.

\section{Understanding Community Needs}

The results of four survey studies on service-delivery in Pakistan (2002 and 2003) suggested providing context-specific family planning services and less costly reproductive health services; confronting women's and men's fears about health side effects of contraception; supplying more widely available and higher quality medical care for postabortion complications; and involving men more effectively in resolving the various problems surrounding unwanted pregnancy (ineffective contraception, induced abortion).

Safe Abortion

Experiences of Women Accessing Legal Abortion in Mexico City, 2008-2009 -High satisfaction with services. -Most women accepted postabortion contraceptive methods. Survey of Health Providers' Opinions of and Experiences with Abortion in Mexico City, 2007-2008

-7 out of 65 interviewed providers were conscientious objectors.

-More than $50 \%$ were in favor of the law. -Participants believed that the acceptability and quality of abortion services had improved over time.

Pilot Study of Contraception and Abortion in Five Country Settings, Pakistan 2007 -One of the main reasons for non-use is non-availability of FP -Men oppose contraception and induced abortion in public, but allow both, if done with confidentiality. -Determination to use contraception increases towards the end of childbearing when there is no desire for more children

\section{Policy}

Access to abortion and postabortion care is highly politicized in many parts of the world where abortion is illegal, as well as where abortion is legal. Local and national government policies often limit a woman's access to sufficient and quality care regarding abortion and PAC. In Mexico, for example, the Council has worked hard to advocate for policies that allow women to seek abortion care. Since the groundbreaking legalization of first-trimester abortion in Mexico City, tens of thousands of women have received safe and legal abortions in the Ministry of Health's (MOH) public health system. To gain a clearer understanding of women's experiences with these services, especially concerning quality of care and access, the Council is finalizing a qualitative study with a small sample of women who accessed legal abortion in the public health system. Further interviews will explore the decisionmaking process and the perceived quality of services. Council researchers also concluded a qualitative study of health providers' opinions of and experiences with the new abortion program (Marieke van Dijk, 2008), yet to be analyzed. 


\section{Access}

Despite the fact that safe, effective and low-resource interventions exist for women who suffer from unsafe abortion and its repercussions, one of the main issues in providing safe abortion and postabortion care is ensuring that all women have sufficient access to the methods and resources (providers, tools, and medications) available. In India, the Council focuses on expanding the MVA provider base through emphasizing the feasibility of abortion by mid-level providers (Shireen Jejeebhoy, Packard Foundation). Efforts to implement new policies that support increased access to comprehensive abortion care (CAC) services and change those policies that restrict safe abortion is a key objective of the Council's work in India (Shveta Kalyanwala, IPAS). Population Council researchers in the area of medical abortion are also investigating how to better expand access to mifepristone in less developed countries as well as developing a highly effective and simple home use medical abortion regimen.

\section{New Technology}

The Council supports WHO reports in concluding that MVA rather than $\mathrm{D \& C}$ is the preferred method of emergency obstetric care due to it effectiveness, acceptability, and low cost (FRONTIERS, 1997).

Researchers have also explored the use of alternative drugs for

\section{Postabortion Care}

FRONTIERS Technical Assistance to Pathfinder for PAC Operations Research, 2005

-Barriers included provider resistance to new techniques (64\%), lack of appropriate service-deliver protocols (40\%), and high demand for services and insufficient staff (40\%).

Linking FP with Postabortion Services in Egypt: Testing feasibility, acceptability, and effectiveness of two models of integration, 2007

$-93 \%$ of physicians on the OB/GYN ward accepted their role in providing FP counseling to PAC patients.

$-77 \%$ of patients actually received FP counseling before discharge

Introducing PAC Services in Rural Senegal, 2000-2002

- Clients were more than twice as likely to report receiving info on FP $-20 \%$ of PAC clients left the health post with a modern contraceptive

prevention and treatment; for example, the Council launched several clinical trials of misoprostol to help identify the best regimen for a number of indications, including treatment of early pregnancy failure.

\section{Service-Delivery}

Throughout many regions of the world, it is the responsibility of the Council to evaluate servicedelivery methods for safe abortion and postabortion care with the implementation of comprehensive monitoring and evaluation programs. In Ghana, a program called Reducing Maternal Mortality and Morbidity (R3M) lowers maternal mortality due to unsafe abortion through reduction in unwanted fertility and provision of comprehensive abortion care (CAC) services. R3M's four activities include increasing the number of quality Health Assistants; providing technical assistance for the development of a CAC and LAPMs training curriculum; developing an M\&E system that is 
integrated with the national District Health Information Management System; and coordinating program partners so that their activities are re-enforcing and cost-effective.

The Council's FRONTIERS program has conducted Operations Research interventions to test the effectiveness and cost-effectiveness of introducing or improving PAC. To address problems of supervision and monitoring specifically, researchers developed innovative computerized, selfanalyzing monitoring and evaluation tools to improve assessment and quality of PAC services (computer-based tools). After reviewing existing PAC supervision instruments in Bolivia, Guatemala and Mexico, FRONTIERS developed an improved model including community and service provider partnerships (Billings and Vernon, 2007).

\section{Integration}

Integrating family planning into postabortion care and reproductive health programs is one of the Council's main goals. A FRONTIERS study in Egypt (Youssef et al, 2007) concluded that both 1) providing family planning counseling and referral to a clinic near the patient's residence and 2) providing family planning counseling and offering a family planning method to postabortion patients were feasible methods of integration. An assessment study of the Senegal PAC program recommended positioning PAC within the health sector reform process to facilitate further institutionalization through transference of funding and technical responsibilities to the central and regional levels, and by incorporation of MVA equipment into the national logistics system (RamaRao et al, 2007).

Several challenges to safe abortion and PAC service accessibility remain in all regions, according to pilot research in sub-Saharan Africa, Latin America, and Asia. These challenges include:

- Establishing sustainable procurement and resupply mechanisms for uterine evacuation instruments, contraceptives, and essential drugs and supplies;

- Improving contraceptive method provision, infection prevention and pain management practices through adequate and continuous training;

- Adequate monitoring and supervision to ensure high-quality, accessible and sustainable services and sufficient numbers of trained mid-level providers;

- Cost of the procedure; and

- Social, religious, policy, and legal restrictions on abortion and contraception.

The ability of reproductive health services to provide high-quality care relies on effective supervision and monitoring, yet supervisors frequently do not know what constitutes high-quality care and how to evaluate services. In order to ensure the long-term effectiveness of safe abortion and PAC programs, qualitative research should focus on an assessment of the opinion and perceived need of local communities and a review of the role of husbands in supporting PAC, FP and counseling.

In the area of postabortion care, more research is needed to provide conclusive data on the best regimens for use of misoprostol, as anecdotal evidence and several qualitative studies indicate that 
there is considerable variation in the ways providers use the drug for a range of gynecologic and postabortion indications. More generally, studies highlight a need to integrate services within the existing health care system, particularly within RH and FP. The Council plans to develop and incorporate accurate assessments of community beliefs in order to change the way safe abortion and PAC providers educate and communicate with their communities about the importance of interpregnancy spacing and use of contraception.

\section{Focused Antenatal Care (FANC)}

In 2001 the WHO issued guidance on a new model of ANC called goal-oriented or focused antenatal care (FANC) for implementation in developing countries, where ANC programs are often poorly implemented and do little to promote the health of mothers and newborns. The new model reduces the number of required antenatal visits to four, and maintains normal pregnancies by identifying existing health conditions, detecting emerging complications, preparing for a healthy birth, and educating clients on postpartum care, including nutrition, breastfeeding, and family planning (WHO 2001). FANC is a comprehensive and integrated service, rather than an aggregation of services that a provider may or may not be able to offer.

FRONTIERS conducted studies in Ghana, Kenya, and South Africa to assess the feasibility, acceptability, and effects of implementing FANC, with regards to the

FANC

Adapting FANC: Lessons from three African countries (Ghana, Kenya, and South Africa), 2008 -Reduction of antenatal visits to four -Heightened efficiency

Developing comprehensive and evidence-based policy and guidelines for antenatal and postnatal care in KwaZulu-Natal, 2008

Acceptability and feasibility of introducing the WHO FANC package in Ghana, 2006

Feasibility of introducing a comprehensive package of antenatal care services in rural public clinics in South Africa, 2005

quality of care provided.

In each intervention, FANC was adapted to the specific needs of the country. Kenya introduced the FANC package with additional components, including PMTCT, intermittent presumptive treatment of malaria, an individual birth plan, TB screening, and comprehensive education. In South Africa, the focused package aimed to integrate into routine ANC service provision of the following: STI prevention and management using syndromic approach; onsite syphilis screening; HIV prevention information, including PMTCT; and active promotion of FP. In Ghana, the program revised RH policy and ANC guidelines to include early detection and treatment of all complications arising during pregnancy, with a particular emphasis on assessing birth preparedness and complication readiness, prevention of malaria in pregnancy and mother-to-child transmission of HIV (PMTCT). 
One of the primary outcomes of the FANC programs was the heightened efficiency and reduction in number of required antenatal visits. The program in South Africa used a comprehensive checklist that consisted of five goal-directed ANC visits and two postnatal care visits. In Ghana, the schedule was reduced from 13 visits to four comprehensive, personalized ANC visits. Guidelines in Kenya specified the appropriate times for providing specific services and education (for example, measuring blood pressure at all visits and discussing family planning at third and fourth visits).

Community orientation manuals were developed to sensitize communities and to enlist the support of opinion leaders, men, and family members during the pregnancy. Staff were typically trained through the cascade approach and included provincial and district health management teams, and providers at the facilities. Job aids (charts and posters) and the integration of services (history taking, lab exams, counseling, etc) helped to ensure that the same provider attended all ANC visits, which appealed to clients because it allowed for more individualized care.

Challenges to the institutionalization of FANC include staff turnover and insufficient involvement of key stakeholders; absence of pre-service training; non-involvement of key informants; limited use of FANC services; funding insufficiencies; and limited implementation. Some clients worried about the longer waiting times and informal fees associated with FANC, even though government policy states that it should be free. More evidence is needed demonstrating the feasibility and effects of integrating FP in to community-based ANC. Other useful indications include collecting evidence from diverse contexts where access to institutional delivery and PNC is very low; longitudinal evidence on the importance of FP information during ANC at different moments of the postpartum period; and evidence on how the amount of FP counseling affects providers and clients. In all regions, the introduction of FANC required training for providers, logistical planning to ensure availability of drugs and supplies, and infrastructure adjustments to change the process of care and to provide a physical space and a functioning laboratory.

\section{ProMPT Ghana: Promoting Malaria Prevention and Treatment}

ProMPT Ghana Promoting Malaria Prevention and Treatment is a three year USAID-funded program under the President's Malaria Initiative (PMI) that was officially launched in March 2009. The program's objectives are to 1) Strengthen the capacity, effectiveness, and reach of Ghana's National Malaria Control Program in reducing the incidence of malaria and its associated morbidity and mortality, and 2) Support the engagement of all key actors in malaria prevention and control, including health workers, non-governmental organizations, schools, communities and the private sector. One component of ProMPT's mandate under PMI is to implement interventions at the facility and community levels to reduce the malaria in pregnancy through proven prevention interventions, thereby addressing two of the most vulnerable populations to malaria: pregnant women and children under five years old. These interventions aim to strengthen Focused Antenatal Care (FANC) and health facility implementation of Intermittent Preventive Treatment of malaria during pregnancy (IPTp) by developing training manuals and job aids, training health workers on malaria in pregnancy, providing equipment and supplies at selected facilities, and revising post supervision tools and follow up visits. 


\section{Safe Delivery}

The Population Council aims to support safe delivery for all women by encouraging the involvement of various types of community-based workers to deliver services directly to women in the home, where the majority of births in developing countries take place. The Council's safe delivery programs focus on the prevention of postpartum hemorrhage (PPH) and preeclampsia/eclampsia (PE/E) — two of the leading causes of maternal mortality worldwide.

\section{Improving Access}

The Population Council's FRONTIERS project supported the scaling up of a community-based model in Kenya that enabled women to give birth safely at home or be referred to a hospital when attended by a skilled midwife from the community. The project demonstrated feasibility, efficacy, and acceptability of the community midwifery approach. Results from one district in Kenya showed a significant increase in home births attended by skilled health workers from $1 \%$ to $9 \%$ between 2001 and 2003.

\section{$\boldsymbol{P P} \boldsymbol{H}^{1}$}

Pilot programs supported by the Council in Ethiopia and Pakistan will examine the community-based distribution of the uterotonic misoprostol for the prevention of

\section{Safe Delivery}

Studies on Magnesium Sulfate Use to Manage Severe PE/E in Mexico, 2009-2011

Operations Research on the use of misoprostol to manage PPH in Pakistan and Ethiopia

Midwifery in Kenya, 2008

-Feasible, effective, and acceptable -Increase in home births attended by skilled health worker, from $1 \%$ to $9 \%$ between 2001 and 2003

PAIMAN, 2004-2009

Global Evaluation of V\&A Interventions, 2009-2012 postpartum hemorrhage. To ensure increased access to care, these programs train community workers to attend to pregnant women throughout the course of pregnancy and to deliver misoprostol immediately after delivery. The Council plans to use the results of the misoprostol pilot programs as a basis for further research and service-delivery in other regions in Africa, Asia, and Latin America. The Council is also providing technical assistance to increase the use, demand, and support of LAPMs and PPH services in Bangladesh.

The Council has provided M\&E support to an USAID-funded bilateral project headed by IntraHealth in Senegal which focuses on improving maternal, newborn and child health outcomes. Through its research and evaluation, the Council has documented the bilateral project's efforts to address postpartum hemorrhage through introducing an evidence-based technique- Active

\footnotetext{
${ }^{1}$ Postpartum hemorrhage occurs during and immediately after delivery and accounts for nearly one quarter of all maternal deaths worldwide (WHO, 2009). The prevailing method for prevention of PPH is Active Management of the Third Stage of Labor (AMSTL), which is simple and inexpensive and can be provided by a trained community worker.
} 
Management of the Third Stage of Labor (AMSTL). Significant improvements have been made with front line providers such as mid-wives feeling more empowered to handle and preempt postpartum hemorrhage. The potential for AMSTL to be included into the MOH protocols and guidelines is considerable.

\section{Pre-Eclampsia/Eclampsia ${ }^{2}$}

In both Nigeria and Mexico, complications due to eclampsia and severe pre-eclampsia are the leading cause of maternal death. Magnesium sulfate is the drug of choice for the management and treatment of these hypertensive disorders; yet it is used infrequently in both countries. The Council has partnered with local ministries of health in both countries to implement a package of advocacy, research and programmatic activities aimed at: 1) drawing attention to the evidence-practice gap; 2) in Nigeria, introducing the use of magnesium sulfate in 10 Kano hospitals; and 3) in Mexico, documenting appropriate use of the drug through a comprehensive review of medical charts of women who suffered severe pre-eclampsia or eclampsia in Mexico City (2005-2007) and the rural Oaxaca state over 2008. In Nigeria, results showed a 40 percent reduction in maternal mortality across the ten sites, hailing MgSO4 a "miracle drug." The contribution of PE/E to maternal mortality was reduced by 68 percent. Work is now underway to transfer activities to government ownership. In Mexico, documented use of magnesium sulfate in both cities was relatively low and inconsistent, despite strong governmental support to promote the correct and consistent use of the drug.

\section{PAIMAN}

The Pakistan Initiative for Mothers and Newborns (PAIMAN) is a five-year project designed to assist the government to reduce maternal, newborn, and child mortality in Pakistan through viable and demonstrable initiatives and capacity-building of existing programs within health systems and communities. Doing so will ensure improvement and supportive links in the continuum of health care for women from the home to the hospital. Specifically, the project aims to increase demand for maternal and child health services; increase use of health care services, particularly antenatal and postnatal care; increase the number of skilled birth attendants and upgrade quality of services; and increase capability and competency of health system budgeting. The Council's specific responsibility is to oversee the management of the monitoring and evaluation component, including Operations Research and knowledge management.

PAIMAN has reached 2.4 million individuals in 10 districts with information about maternal and newborn health care issues. Thirty one public sector health facilities have been upgraded and reequipped to provide 24/7 Emergency Obstetric and Newborn Care Services in 10 districts. Eighteen

\footnotetext{
${ }^{2}$ Pre-eclampsia/eclampsia is of unknown etiology and the definitive treatment is delivery itself. The World Health Organization has recommended magnesium sulfate as the most effective, safe, and low-cost drug to prevent and manage severe pre-eclampsia and eclampsia.
} 
hundred public sector clinics have been established to provide care for poor urban populations and 70,000 clean delivery kits have been distributed in the community. Expected outcomes of the program include reduced neonatal mortality rates and increased proportion of live births assisted by SBA. More information can be found at the program's website: http://www.paiman.org.pk/.

\section{Voucher-and-Accreditation Interventions}

Once intervention programs have been proven effective for promoting safe delivery, problems remain in ensuring their funding and long term sustainability. The Council is undertaking a series of public health evaluations of a number of alternative financing schemes, such as Voucher and Accreditation programs, in order to generate evidence to help governments decide whether to scale up these programs, include additional services, or support their transition to a different business model. The Council's evaluations will take place in five countries (Bangladesh, Cambodia, Kenya, Tanzania, and Uganda) under a five-year project. So far Voucher and Accreditation programs have proved to be quite effective in increasing antenatal care, skilled attendance at birth and less effective with family planning. As of yet, it is uncertain who benefits from these schemes, whether they increase quality of care, and clarity is needed on the type of performance incentives to give providers.

The main challenges to promoting safe delivery programs are: 1) how to improve access to people, and 2) how to improve staff and ensure supplies within the health system. These goals are contingent on strong and consistent linkages to the formal health system, communication and transport for referral, reliable sources of supplies and equipment, community involvement and support, and service charges for the poor.

\section{Postpartum Care}

Most maternal and newborn deaths occur very soon after delivery-over 60 percent of maternal deaths occur in the first 48 hours after childbirth (WHO 2005). For many women, particularly in eastern and southern Africa, the postpartum period is a time of increased susceptibility to HIV and STIs. Providing continuity of care between antenatal care services, prevention of mother-to-child transmission of HIV, delivery, and postpartum care can ensure that women's fertility intentions in the context of their HIV status are fully and effectively met, while at the same time offering opportunities to address more direct causes of maternal morbidity and mortality, such as bleeding and infection. 
FRONTIERS in Kenya developed and introduced a strengthened postnatal care package for women, including four postnatal consultations (within 48 hours and at two weeks, six weeks, and six months). Messages on healthy timing and spacing of pregnancies have been incorporated into training and job aids.

In Lesotho, the Council is involved in a project designed to address the rapid rise in HIV incidence among antenatal clients. The intervention tests the feasibility and effects of a strengthened postpartum care program, which includes syphilis screening and management; HIV testing, counseling, and PMTCT; discussion of postpartum family planning choices; and discussion of prevention of sexually transmitted infections, including HIV. The approach includes three postpartum visits — at 24 hours, one week, and six weeks. Additionally, in Swaziland a Population Council project demonstrated that high-quality integrated PMTCT programs and MNH postnatal services are feasible and acceptable, and can result in promoting early postnatal visits and improved care of both HIV-positive and HIVnegative mothers and their babies (Charlotte Warren, 2009).

\section{Family planning}

Postpartum care not only aims to reduce maternal and newborn mortality by targeting infection and bleeding, but also by promoting continual family planning. In Egypt, the Council is involved in a project to assist the government in integrating birth-spacing and family planning messages into antenatal and postpartum services to help promote healthy birth intervals and avoid unplanned pregnancies in the future.

Although Pakistan was among the first countries in the world to implement a national family planning program, use of contraception has reached neither the levels hoped for, nor the levels achieved in many other developing countries. Family Advancement for Life and Health (FALAH) is a five-year undertaking to diversify family planning services in Pakistan by working to remove barriers; improve understanding of the value of FP; increase knowledge of birth spacing methods; and improve access to and quality of care in both the public and private sectors. By December 2009, more than half a million people (352,406 men and 218,232 women) had been contacted by FALAH for FP orientation through neighborhood meetings. Nearly 24,000 women of reproductive age were given free consultations on FP at 1,178 mobile clinics. More than 16,000 men and women ("influencers") have been mobilized for community outreach through orientation meetings. And 
221,225 women were contacted through household visits. Many of these consultations and outreach efforts were aimed at promoting FP during the postpartum period.

The main challenge of promoting postpartum care is integrating preventive care for postpartum health risks within ANC and general RH programs. Additionally, encouraging family planning in the period directly after delivery is difficult, particularly due to myths and fears of family planning methods. Safe motherhood services do not end with a woman's delivery. Postpartum care is crucial to reduce global maternal and newborn morbidity and mortality.

\section{Conclusion}

Population Council policy for safe motherhood includes quality of care, gender sensitivity and male involvement, education and training (using Information Education and Communication materials), integration, monitoring and evaluation, and efforts to improve access to services. These policies and goals are incorporated in every stage of the cycle of pregnancy, including safe abortion and postabortion care, antenatal care, safe delivery, and postpartum care. 